\title{
ON A RECURRENCE FORMULA FOR ELEMENTARY SPHERICAL FUNCTIONS ON SYMMETRIC SPACES AND ITS APPLICATIONS TO MULTIPLIERS FOR THE SPHERICAL FOURIER TRANSFORM
}

\section{LARS VRETARE}

\section{Introduction.}

Consider spherical functions on a symmetric space, the spherical Fourier transform $\hat{f}$ and its inverse $\breve{F}$. $F$ is said to be a $L_{p}$ Fourier multiplier if the mapping $f \rightarrow \breve{F} * f$ is a bounded operator on $L_{p}$. Denote by $m_{p}$ the set of all such multipliers, and put

$$
\|F\|_{m_{p}}=\sup _{0 \neq f \in L_{p}} \frac{\|\check{F} * f\|_{p}}{\|f\|_{p}}
$$

i.e. the corresponding operator norm.

In the papers [2] and [3] sufficient conditions on $F$ to be a multiplier were obtained in some special cases of symmetric spaces. [2] was concerned with the case of a compact group and [3] with the noncompact real rank one and complex cases. In both papers an important role was played by a recurrence formula for the elementary spherical functions $\varphi_{\lambda}$

$$
\omega^{L} \varphi_{\lambda}=\sum_{j=1}^{q} c_{j}(\lambda) \varphi_{\lambda-i \sigma_{j}}
$$

In [4] the validity of such a formula in the general case was confirmed. The purpose of this paper is to derive regularity conditions for the coefficients $c_{j}(\lambda)$ in order to extend the multiplier theorems to general compact and noncompact symmetric spaces.

The paper is divided into four sections. In section 1 we explain the notations and begin the investigations of the coefficients while section 2 is devoted to a closer study. The application to multipliers in the noncompact case is made in section 3 and finally in section 4 we briefly scetch how to treat the compact case.

Received September 29, 1976. 


\section{Preliminaries.}

Let $G=K A N$ be an Iwasawa decomposition of a connected, non-compact, semisimple Lie group over $R$, with finite centre, and let $\mathfrak{g}_{0}=\mathfrak{f}_{0}+\mathfrak{h}_{\mathfrak{p}_{0}}+\mathfrak{n}_{0}$ be the corresponding decomposition of its Lie algebra. Denote by $\Delta_{0}^{+}$the positive root system of the pair $\left(\mathfrak{g}_{0}, \mathrm{~h}_{\mathfrak{p}_{0}}\right)$ and by $\varrho$ the half-sum of the restricted roots. The elementary spherical functions on $G / K$ are defined by

$$
\varphi_{\lambda}(g)=\int_{K} e^{(i \lambda-\varrho)(H(g k))} d k, \quad \lambda \in \mathfrak{h}_{\mathfrak{p}_{0}}^{*}+i \mathfrak{h}_{\mathfrak{p}_{0}}^{*}
$$

where $g=k \exp H(g) n$, and $\mathfrak{h}_{\mathfrak{p}_{0}}^{*}$ is the real dual of $\mathfrak{h}_{\mathfrak{p}_{0}}$. Let us identify $\mathfrak{h}_{\mathfrak{p}_{0}}$ and $\mathfrak{h}_{\mathfrak{p}_{0}}^{*}$ under the scalar product $\langle\cdot, \cdot\rangle$ on $\mathfrak{h}_{\mathfrak{p}_{0}}$, given by the Killing form, and let us also write $\mathfrak{h}_{\mathfrak{p}}$ for the complexification of $\mathfrak{h}_{\mathfrak{p}_{0}}$. The spherical Fourier transform and its inverse are formally defined by

$$
\hat{f}(\lambda)=\int_{G} f(g) \varphi_{-\lambda}(g) d g
$$

and

$$
\check{F}(g)=\int_{\mathfrak{h}_{\mathfrak{p}_{0}}} F(\lambda) \varphi_{\lambda}(g) \frac{d \lambda}{|c(\lambda)|^{2}}
$$

where $c(\lambda)$ is Harish Candra's $c$-function.

Fix a $\sigma \in \mathfrak{h}_{\mathfrak{p}_{0}}$ such that $\langle\sigma, \alpha\rangle /\langle\alpha, \alpha\rangle$ is a nonnegative integer for all $\alpha \in \Delta_{0}^{+}$. As was proved in [4], we can find a finite number of $\sigma_{j}^{\prime} \in \mathfrak{h}_{\mathfrak{p}_{0}}$ and rational functions $c_{j}^{\prime}(\lambda)$ such that

$$
\varphi_{-i(\sigma+\varrho)}(g) \varphi_{\lambda}(g)=\sum_{j} c_{j}^{\prime}(\lambda) \varphi_{\lambda-i \sigma_{j}^{\prime}}(g)
$$

Also, because of the relation $\varphi_{\lambda}\left(g^{-1}\right)=\varphi_{-\lambda}(g)$

$$
\varphi_{-i(\sigma+\varrho)}\left(g^{-1}\right) \varphi_{\lambda}(g)=\sum_{j} c_{j}^{\prime}(-\lambda) \varphi_{\lambda+i \sigma_{j}^{\prime}}(g) .
$$

Let us now define the function $\omega$ by putting

$$
\omega(g)=\varphi_{-i(\sigma+\varrho)}(g)+\varphi_{-i(\sigma+e)}\left(g^{-1}\right)-2 .
$$

Iteration of the two formulas above yields

LEMma 1.1. Let $L$ be a fixed positive integer. There exist $\sigma_{1}, \ldots, \sigma_{q}$ in $\mathfrak{h}_{\mathfrak{p}_{0}}$ and rational functions $c_{1}(\lambda), \ldots, c_{q}(\lambda)$ such that

$$
\omega^{L} \varphi_{\lambda}=\sum_{j=1}^{q} c_{j}(\lambda) \varphi_{\lambda-i \sigma_{j}}
$$


Let $X_{1}, \ldots, X_{n}$ be a base for $\mathfrak{g}_{0}$ and let for any positive integral $n$-tuple $M=\left(m_{1}, \ldots, m_{n}\right), X(M)$ be the differential operator corresponding to the coefficient of $t^{M}$ in

$$
\frac{1}{|M| !}\left(\sum_{i=1}^{n} t_{i} X_{i}\right)^{|M|}
$$

that is,

$$
X(M) f(g)=\frac{1}{M !}\left\{D^{M} f\left(g \exp \sum t_{i} X_{i}\right)\right\}_{t=0} .
$$

Lemma 1.2. Let $e$ be the unit of $G$. Then

$$
X(M) \omega^{L}(e)=0 \quad \text { if }|M|<2 L
$$

and

$$
\left|X(N) \varphi_{\lambda}(e)\right| \leqq C(1+|\lambda|)^{|N|}, \quad \lambda \in \mathfrak{h}_{\mathfrak{p}} .
$$

Proof. A direct application of the definitions of $X(M)$ and $\omega$ respectively proves the first statement for $L=1$. The general case is then obvious. The second statement is equivalent to lemma 46 in [1], although the degree $|N|$ is not specifically mentioned.

The Casimir operator of $\mathfrak{g}_{0}$ is defined by $A=\sum a_{i j} X_{i} X_{j}$ where $\left(a_{i j}\right)$ is the inverse of the matrix $\left(\left\langle X_{i}, X_{j}\right\rangle\right)$. In order to apply the operator $A^{l}$ to the function $\omega^{L} \varphi_{\lambda}$, we write

$$
A^{l}=\sum_{|I| \leqq 2 l} a_{I} X(I)
$$

and

$$
\dot{X}(I) \omega^{L} \varphi_{\lambda}=\sum_{M+N=I} X(M) \omega^{L} X(N) \varphi_{\lambda}
$$

Corollary 1.3. Let $A$ be the Casimir operator of $\mathfrak{g}_{0}$. Then

$$
A^{l} \omega^{L} \varphi_{\lambda}(e)=0 \quad \text { if } l<L
$$

and

$$
\left|A^{l} \omega^{L} \varphi_{\lambda}(e)\right| \leqq C(1+|\lambda|)^{2 l-2 L} \quad \text { if } l \geqq L
$$

Now, let us also compute $A^{l} \omega^{L} \varphi_{\lambda}(e)$ from the recurrence formula. $\varphi_{\lambda}$ is an eigenfunction of $A$ with eigenvalue $\chi(\lambda)=-\langle\lambda, \lambda\rangle-\langle\varrho, \varrho\rangle$, and $\varphi_{\lambda}(e)=1$. 
Consequently

$$
A^{l} \omega^{L} \varphi_{\lambda}(e)=\sum_{j=1}^{q} c_{j}(\lambda)\left[\chi\left(\lambda-i \sigma_{j}\right)\right]^{l} .
$$

Also, by the binomial theorem

$$
\sum_{j=1}^{q} c_{j}(\lambda)\left(\chi\left(\lambda-i \sigma_{j}\right)-\chi(\lambda)\right)^{k}=\sum_{l=0}^{k}(-1)^{k-l}\left(\begin{array}{c}
k \\
l
\end{array}\right)(\chi(\lambda))^{k-l} A^{l} \omega^{L} \varphi_{\lambda}(e) .
$$

This proves the first part of

THEOREM 1.4. The coefficients in the recurrence formula

$$
\omega^{L} \varphi_{\lambda}=\sum_{j=1}^{q} c_{j}(\lambda) \varphi_{\lambda-i \sigma_{j}}
$$

satisfy the following two conditions

1)

$$
\left|\sum_{j=1}^{q} c_{j}(\lambda) \chi\left(\lambda-i \sigma_{j}\right)-\chi(\lambda)^{k}\right| \leqq C(1+|\lambda|)^{2 k-2 L}
$$

for all $\lambda \in \mathfrak{h}_{\mathfrak{p}}$ if $k \geqq L$, and it is equal to zero if $0 \leqq k<L$.

2)

$$
\left|c_{j}(\lambda)\right| \leqq C \prod_{\alpha \in \Delta_{0}^{+}} \frac{1+|\langle\alpha, \lambda\rangle|}{|\langle\alpha, \lambda\rangle|} \quad \text { if } \lambda \in \mathfrak{h}_{\mathfrak{p}_{0}} .
$$

The proof of condition 2) will be given as a series of lemmas in the next section.

\section{Further investigation of the coefficients $c_{j}(\lambda)$.}

The ideas of this section, leading to a proof of condition 2) of theorem 1.4, are as follows. First we consider the denominator of $c_{j}(\lambda)$. It turns out that $\prod_{\alpha \in \Delta_{0}^{+}}\langle\alpha, \lambda\rangle c_{j}(\lambda)$ is nonsingular on $\mathfrak{h}_{\mathfrak{p}_{0}}$. Next we prove that $c_{j}(\lambda)$ is bounded if $\operatorname{Im} \lambda$ is keeping away from the walls of the Weyl chambers in $\mathfrak{h}_{\mathfrak{p}_{0}}$. Finally the bound on $\mathfrak{h}_{\mathfrak{p}_{0}}$ will follow by means of classical complex analysis.

Thus we start by asking for an explicit expression for the denominator of $c_{j}(\lambda)$. To this end we solve the system of equations obtained from (1.1) by putting $l=1, \ldots, q$.

LEMMA 2.1. The rational functions $c_{j}(\lambda)$ are given by

$$
c_{j}(\lambda)=\sum_{l=1}^{q} \varepsilon_{j l}(\lambda) A^{l} \omega^{L} \varphi_{\lambda}(e)
$$


where $\varepsilon_{j l}$ is the coefficient of $z^{l}$ in the polynomial

$$
\prod_{\substack{k=1 \\ k \neq j}}^{q}\left(z-\chi_{k}\right)\left(\chi_{j}-\chi_{k}\right)^{-1}, \quad \chi_{k}=\chi\left(\lambda-i \sigma_{k}\right) .
$$

Since $A^{l} \omega^{L} \varphi_{\lambda}(e)$ is a polynomial in $\lambda$, we find the denominator of $c_{j}(\lambda)$ to be

$$
\prod_{\substack{k=1 \\ k \neq i}}^{q}\left(\chi_{j}-\chi_{k}\right)
$$

Then what is the multiplicity of these factors?

Lemma 2.2. The singularities of $c_{j}(\lambda)$ are all simple.

Proof. Suppose that two factors are proportional e.g. $\chi_{j}-\chi_{k}=B\left(\chi_{j}-\chi_{l}\right)$, $j, k, l$ all different. Simplifications leads to

$$
2 i\left\langle\lambda, \sigma_{k}-(1-B) \sigma_{j}-B \sigma_{l}\right\rangle+\left\langle\sigma_{k}, \sigma_{k}\right\rangle-(1-B)\left\langle\sigma_{j}, \sigma_{j}\right\rangle-B\left\langle\sigma_{l}, \sigma_{l}\right\rangle=0
$$

for all $\lambda \in \mathfrak{h}_{\mathfrak{p}}$, i.e.

$$
\left\{\begin{array}{l}
\sigma_{k}=(1-B) \sigma_{j}+B \sigma_{l} \\
\left\langle\sigma_{k}, \sigma_{k}\right\rangle=(1-B)\left\langle\sigma_{j}, \sigma_{j}\right\rangle+B\left\langle\sigma_{l}, \sigma_{l}\right\rangle
\end{array}\right.
$$

Squaring the first equation and then subtracting the second one we get

$$
0=B(B-1)\left\langle\sigma_{j}-\sigma_{l}, \sigma_{j}-\sigma_{l}\right\rangle .
$$

But this is a contradiction since $\sigma_{j}, \sigma_{k}$ and $\sigma_{l}$ are all different.

It turns out to be more convenient in the sequel to consider the function

$$
d_{j}(\lambda)=c_{j}(\lambda) \frac{c\left(\lambda-i \sigma_{j}\right)}{c(\lambda)}
$$

rather than $c_{j}(\lambda)$ itself. Note that $c\left(\lambda-i \sigma_{j}\right) / c(\lambda)$ is a product of functions of the type

$$
\frac{\langle i \lambda, \alpha\rangle+x}{\langle i \lambda, \alpha\rangle+y}
$$

where $x$ and $y$ are real numbers and $\alpha \in \Delta_{0}^{+}$.

Lemma 2.3. The following symmetry relation holds.

$$
d_{j}(\lambda)=d_{j}\left(-\lambda+i \sigma_{j}\right) .
$$


Proof. Let $C_{\varrho}$ denote the convex hull of the images of $\varrho$ under the Weyl group $W$, and put $F_{\varepsilon}=\mathfrak{h}_{\mathfrak{p}_{0}}+i \varepsilon C_{\varrho}$. Fix an $\varepsilon>0$ such that the points $i \sigma_{1}, \ldots, i \sigma_{q}$ all belong to the tube $F_{\varepsilon}$. In view of well-known properties of $\varphi_{\lambda}$ and the $c$ function it is easy to see that it is possible to find a $W$-invariant polynom $p(\lambda)$ such that

$$
p(\lambda) c_{j}(\lambda) \varphi_{\lambda-i \sigma_{j}} \frac{1}{c(\lambda) c(-\lambda)}
$$

is holomorphic in $F_{\varepsilon}$ and bounded by a polynomial for all $j$. Now for any $W$ invariant function $\hat{f}^{\prime}$, which is rapidly decreasing and holomorphic in the tube $F_{\varepsilon}$, put $\hat{f}=p \hat{f}^{\prime}$. Due to our construction the following application of Cauchy's theorem is legitime

$$
\begin{aligned}
\omega^{L} f & =\omega^{L} \int_{\mathfrak{h}_{\mathfrak{p}_{0}}} \hat{f}(\lambda) \varphi_{\lambda} \frac{d \lambda}{|c(\lambda)|^{2}}=\sum_{j=1}^{q} \int_{\mathfrak{h}_{\mathfrak{p}_{0}}} \hat{f}(\lambda) c_{j}(\lambda) \varphi_{\lambda-i \sigma j} \frac{d \lambda}{|c(\lambda)|^{2}} \\
& =\sum_{j=1}^{q} \int_{\mathfrak{h}_{\mathfrak{p}_{0}}} \hat{f}\left(\lambda+i \sigma_{j}\right) c_{j}\left(\lambda+i \sigma_{j}\right) \varphi_{\lambda} \frac{d \lambda}{c\left(\lambda+i \sigma_{j}\right) c\left(-\lambda-i \sigma_{j}\right)} .
\end{aligned}
$$

We conclude that

$$
\left(\omega^{L} f \hat{)}(\lambda)=\sum_{j=1}^{q} \hat{f}\left(\lambda+i \sigma_{j}\right) c_{j}\left(\lambda+i \sigma_{j}\right) \frac{c(\lambda) c(-\lambda)}{c\left(\lambda+i \sigma_{j}\right) c\left(-\lambda-i \sigma_{j}\right)} .\right.
$$

But we also have more directly

$$
\left(\omega^{L} f\right) \hat{(\lambda)}=\int_{G} \omega^{L}(g) f(g) \varphi_{-\lambda}(g) d g=\sum_{j=1}^{q} c_{j}(-\lambda) \hat{f}\left(\lambda+i \sigma_{j}\right) .
$$

Comparison of these two expressions for $\left(\omega^{L} f\right)^{\wedge}$ shows that

$$
c_{j}(-\lambda)=\frac{c(\lambda) c(-\lambda)}{c\left(\lambda+i \sigma_{j}\right) c\left(-\lambda-i \sigma_{j}\right)} c_{j}\left(\lambda+i \sigma_{j}\right)
$$

or equivalently

$$
d_{j}(-\lambda)=d_{j}\left(\lambda+i \sigma_{j}\right)
$$

Lemma 2.4. $c_{j}(\lambda) \prod_{\alpha \in \Delta_{0}^{+}}\langle\alpha, \lambda\rangle$ is nonsingular on $\mathfrak{h}_{\mathfrak{p}_{0}}$.

Proof. By lemma 2.1 the denominator of $c_{j}(\lambda) \prod_{\alpha \in \Delta_{0}^{+}}\langle\alpha, \lambda\rangle$ factors into firstorder polynomials $\langle i \lambda, v\rangle+a$, where $v \in \mathfrak{h}_{\mathfrak{p}_{0}}$ and $a$ is a real number. According to lemma 2.2 all these factors have unit multiplicity so the only possible singularities are when $v$ is not a root. Let $r(\lambda)$ be such a factor. We have to show that $r(\lambda)$ is nonzero on $\mathfrak{h}_{\mathfrak{p}_{0}}$. Now, since $v$ is not a root $r(\lambda)$ must be 
a factor of $d_{j}(\lambda)$, and lemma 2.3 shows that $r\left(-\lambda+i \sigma_{j}\right)$ is also a factor of $d_{j}(\lambda)$ and $c_{j}(\lambda)$. But then we can write

$$
r\left(-\lambda+i \sigma_{j}\right)=C\left(\chi\left(\lambda-i \sigma_{j}\right)-\chi\left(\lambda-i \sigma_{k}\right)\right)
$$

for some $k \neq j$, or

$$
\begin{aligned}
r(\lambda) & =C\left(\chi(-\lambda)-\chi\left(-\lambda+i \sigma_{j}-i \sigma_{k}\right)\right) \\
& =C\left(2 i\left\langle\lambda, \sigma_{j}-\sigma_{k}\right\rangle+\left\langle\sigma_{j}-\sigma_{k}, \sigma_{j}-\sigma_{k}\right\rangle\right) .
\end{aligned}
$$

This shows that $r(\lambda)$ is nonzero on $\mathfrak{h}_{\mathfrak{p}_{0}}$, and the proof is finished.

Consider now the restriction to $\mathfrak{h}_{\mathfrak{p}}$ of the function $\omega^{\mathrm{L}}$. This is a finite linear combination of exponentials $e^{\tau_{l}}, l=1, \ldots, p$, where $\left\langle\tau_{l}, \alpha\right\rangle\langle\langle\alpha, \alpha\rangle$ is an integer for all $\alpha \in \Delta_{0}^{+}$and all $l$. We shall also make use of Harish Chandra's expansion for elementary spherical functions.

$$
\varphi_{\lambda}=e^{-e} \sum_{S \in W} c(S \lambda) \Phi_{S \lambda}
$$

where

$$
\Phi_{\lambda}=e^{i \lambda} \sum_{\mu \in E} \Gamma_{\mu}(\lambda) e^{-\mu}
$$

and $E$ denotes the lattice over the nonnegative integers spanned by the simple roots in $\Delta_{0}^{+}$. Expanding both sides of the recurrence formula and equating the coefficients of $e^{\sigma_{j}-\varrho+i \lambda}$ (cf. [1, p. 307]) we obtain

$$
\sum_{l=1}^{p} a_{l} \Gamma_{\tau_{l}-\sigma_{j}}(\lambda) c(\lambda)=\sum_{k=1}^{q} c_{k}(\lambda) \Gamma_{\sigma_{k}-\sigma_{j}}\left(\lambda-i \sigma_{k}\right) c\left(\lambda-i \sigma_{k}\right)
$$

where we have put $\Gamma_{\mu}=0$ if $\mu \notin E$. Note also that $\Gamma_{0}=1$. This formula serves to define $c_{j}(\lambda)$ recursively. More precisely, by introducing in $\mathfrak{h}_{\mathfrak{p}_{0}}$ lexiocographic ordering with respect to the simple roots we can make the following reformulation.

Lemma 2.5. The functions $d_{j}$ are recursively determined by

$$
d_{j}(\lambda)=\sum_{l=1}^{p} a_{l} \Gamma_{\tau_{l}-\sigma_{j}}(\lambda)-\sum_{\sigma_{k}>\sigma_{j}} d_{k}(\lambda) \Gamma_{\sigma_{k}-\sigma_{j}}\left(\lambda-i \sigma_{k}\right) .
$$

For any $M \geqq 0$ let $\mathfrak{h}_{\mathfrak{p}_{0}}(M)$ be the subset of $\mathfrak{h}_{\mathfrak{p}_{0}}$ for which $\langle\alpha, h\rangle>M$ for all $\alpha \in \Delta_{0}^{+}$.

COROLLARY 2.6. There exist a number $M$ such that all $d_{j}$ are bounded on $\mathfrak{h}_{\mathfrak{p}_{0}}$ $-i \mathfrak{h}_{\mathfrak{p}_{0}}(M)$. 
Proof. It is sufficient to choose $M$ such that $\Gamma_{\mu}(\lambda)$ and $\Gamma_{\mu}\left(\lambda-i \sigma_{j}\right)$ are bounded on $\mathfrak{h}_{\mathfrak{p}_{0}}-i \mathfrak{h}_{\mathfrak{p}_{0}}(M)$ for all $\mu \in E$ and all $j$. Since $\Gamma_{\mu}(v)$ is bounded on $\mathfrak{h}_{\mathfrak{p}_{0}}$ $-i \mathfrak{h}_{\mathfrak{p}_{0}}(0)$, see [5, p. 334], this holds if $M+\left\langle\alpha, \sigma_{j}\right\rangle>0$ for all $\alpha \in \Delta_{0}^{+}$and all $j$.

Returning to $c_{j}$ we get in view of the $W$-invariance of the recurrence formula

Corollary 2.7. It is possible to choose $M$ such that $c_{j}$ is bounded on $\mathfrak{h}_{\mathfrak{p}_{0}}$ $-i S \mathfrak{h}_{\mathfrak{p}_{0}}(M)$ for all $S \in W$, especially on $\mathfrak{h}_{\mathfrak{p}_{0}} \pm i \mathfrak{h}_{\mathfrak{p}_{0}}(M)$.

We also need

Lemma 2.8. Let $f(\lambda)$ be a rational function defined on $\mathfrak{h}_{\mathfrak{p}}$, bounded by a constant $C$ on $\mathfrak{h}_{\mathfrak{p}_{0}} \pm$ in for some $\eta \in \mathfrak{h}_{\mathfrak{p}_{0}}$ and analytic in the tube $|\operatorname{Im} \lambda| \leqq|\eta|$. Then $f(\lambda)$ is bounded by the same constant $C$ on $\mathfrak{h}_{\mathfrak{p}_{0}}$.

Proof. For fixed $\xi, \eta \in \mathfrak{h}_{\mathfrak{p}_{0}}, f(\xi+z \eta)$ is a rational function of one complex variable $z$. By assumption it is bounded by $C$ on the lines $\operatorname{Im} z= \pm 1$ and analytic in the strip $|\operatorname{Im} z| \leqq 1$. Being rational it must be analytic at infinity hence bounded by $C$ in the whole strip.

We are now ready to prove condition 2) of theorem 1.4. As we have seen the denominator of $c_{j}(\lambda)$ may be written

$$
\prod_{k \neq j}\left(\left\langle i \lambda, v_{k}\right\rangle+a_{k}\right)
$$

Multiplication of $c_{j}(\lambda)$ by

$$
h(\lambda)=\prod_{k \neq j} \frac{\left\langle i \lambda, v_{k}\right\rangle+a_{k}}{\left\langle i \lambda, v_{k}\right\rangle+D}
$$

will therefore remove the singularities without changing the bound on $\mathfrak{h}_{\mathfrak{p}_{0}}(M)$. More precisely, if $\eta \in \mathfrak{h}_{\mathfrak{p}_{0}}(M)$ is kept fixed and $D$ is choosen sufficiently large the rational function $f(\lambda)=h(\lambda) c_{j}(\lambda)$ will be analytic in the tube $|\operatorname{Im} \lambda| \leqq|\eta|$ and bounded if $\operatorname{Im} \lambda= \pm \eta$. We conclude from lemma 2.8 that $f$ is bounded on $\mathfrak{h}_{\mathfrak{p}_{0}}$ as well. To get back to $c_{j}(\lambda)$ recall that the denominators of $1 / h(\lambda)$ and $c_{j}(\lambda)$ are identical. Lemma 2.4 then shows that

$$
\prod_{\alpha \in \Delta_{0}^{+}} \frac{|\langle\alpha, \lambda\rangle|}{1+|\langle\alpha, \lambda\rangle|} \frac{1}{h(\lambda)}
$$

is bounded on $\mathfrak{h}_{\mathfrak{p}_{0}}$ and multiplication by $f(\lambda)$ yields the desired result

$$
\prod_{\alpha \in \Delta_{0}^{+}} \frac{|\langle\alpha, \lambda\rangle|}{1+|\langle\alpha, \lambda\rangle|} \cdot\left|c_{j}(\lambda)\right| \leqq C, \quad \lambda \in \mathfrak{h}_{\mathfrak{p}_{0}} .
$$




\section{Multipliers.}

A $W$-invariant function $F$ on $\mathfrak{h}_{\mathfrak{p}_{0}}$ is called a $L_{p}$ Fourier multiplier if the mapping $f \rightarrow \breve{F} * f$ extends to a bounded operator on $L_{p}=L_{p}(K \backslash G / K)$. Following [3] we shall now derive estimates for the multiplier or $m_{p}$ norm of $F$, i.e. the corresponding operator norm. We restrict ourselves to radial multipliers, $F(\lambda)=u(\chi(\lambda))$. Recall that $\chi$ is the eigenvalue of the Casimir operator, $\chi(\lambda)=-\langle\lambda, \lambda\rangle-\langle\varrho, \varrho\rangle$.

An application of Schwarz' inequality to $\|\check{F}\|_{1}$ yields in the usual way

$$
\|F\|_{m_{p}} \leqq C\|\check{F}\|_{2}^{1-n / 4 L}\left\|\omega^{L} \check{F}\right\|_{2}^{n / 4 L}+C\left\|\omega^{L} \check{F}\right\|_{2}
$$

where $L$ is a sufficiently large integer and $n=\operatorname{dim} G / K$. The $L_{2}$ norms are handled by Parseval's formula

$$
\int_{G}|f(g)|^{2} d g=\int_{\mathfrak{h}_{\mathfrak{p}_{0}}}|\hat{f}(\lambda)|^{2} \frac{d \lambda}{|c(\lambda)|^{2}}
$$

and under certain conditions on $u$, which will be made more precise later, the spherical Fourier transform of $\omega^{L} \check{F}$ can be computed by means of the recurrence formula. Hence

$$
\left\|\omega^{L} \check{F}\right\|_{2}=\left\|\sum_{j=1}^{q} c_{j}(\lambda) u\left(\chi\left(\lambda-i \sigma_{j}\right)\right)\right\|_{2}
$$

The use of equal notation for different $L_{2}$ norms will cause no confusion. In [3] the sum to the right was first expressed in terms of differences of $u$ and then each difference was estimated by the corresponding derivative. We now proceed more directly using Taylor's formula for $u$ at the point $\chi(\lambda)$. Thus

$$
\begin{aligned}
&\left|\left(\omega^{L} \check{F}\right)^{\hat{\gamma}}(\lambda)\right| \leqq C \sum_{k=0}^{2 L-1}\left|u^{(k)}(\chi(\lambda))\right|\left|\sum_{j=1}^{q} c_{j}(\lambda)\left(\chi\left(\lambda-i \sigma_{j}\right)-\chi(\lambda)\right)^{k}\right|++ \\
&+C \sum_{j=1}^{q}\left|c_{j}(\lambda)\right|\left|\chi\left(\lambda-i \sigma_{j}\right)-\chi(\lambda)\right| 2 L \sup _{\xi \in \Omega_{\lambda}}\left|u^{(2 L)}(\xi)\right|
\end{aligned}
$$

where $\Omega_{\lambda}$ is the convex hull of the points $\chi(\lambda)$ and $\chi\left(\lambda-i \sigma_{j}\right), j=1, \ldots, q$. If we now divide both sides by the $c$-function and make use of theorem 1.4, together with the fact that

$$
\prod_{\alpha \in \Delta_{0}^{+}} \frac{1+|\langle\alpha, \lambda\rangle|}{|\langle\alpha, \lambda\rangle|} \cdot \frac{1}{|c(\lambda)|} \leqq C(1+|\lambda|)^{m / 2}, \quad \lambda \in \mathfrak{h}_{\mathfrak{p}_{0}}
$$

where $m=\operatorname{dim} G / K-\operatorname{rank} G / K$, we obtain

LEMMA 3.1. Under suitable conditions on $u$ the following estimate holds on $\mathfrak{h}_{\mathfrak{p}_{0}}$ 


$$
\begin{aligned}
\frac{1}{|c(\lambda)|}\left|\left(\omega^{L} \check{F}\right)^{\gamma}(\lambda)\right| \leqq C \sum_{k=L}^{2 L-1}(1+|\lambda|)^{2 k-2 L+m / 2}\left|u^{(k)}(\chi(\lambda))\right|+ \\
+C(1+|\lambda|)^{2 L+m / 2} \sup _{\xi \in \Omega_{\lambda}}\left|u^{(2 L)}(\xi)\right|
\end{aligned}
$$

From now on we consider multipliers $u\left(\chi(\lambda) / N^{2}\right)$, depending on a parameter $N$ which is assumed to be large. Let us write

$$
u_{N}(z)=u\left(\frac{z}{N^{2}}\right) \text {. }
$$

It is clear that if $u(z), z=x+i y$, is holomorphic in the interior of a parabola $y^{2}$ $=a(x+b), a>0, b>0$, and if $N$ is sufficiently large then $u_{N}(\chi)$ is holomorphic in any given tube centred at $\mathfrak{h}_{\mathfrak{p}_{0}}$. We call such a region a parabolic neighbourhood of the positive real axis or shorter a p.n. If also

$$
\sup _{\text {p.n. }}|z|^{j}\left|u^{(k)}(z)\right|<\infty
$$

for all $k, j=0,1, \ldots$ then $u_{N}(\chi)$ is rapidly decreasing in the tube and lemma 3.1 will hold.

Here is our first result

THEOREM 3.2. Let $u(z)$ be holomorphic in a p.n. and suppose that

$$
\sup _{\text {p.n. }}|z|^{j}\left|u^{(k)}(z)\right|<\infty
$$

for all $k, j=0,1, \ldots$ Then there is a number $N_{0}$ such that $\left\|u_{N}(\chi)\right\|_{m_{p}}$ is uniformly bounded for $N \geqq N_{0}$.

Proof. Put

$$
\left\|u_{N}\right\|_{W_{2}^{L}}=\left\|\omega^{L}\left(u_{N}(\chi)\right)^{\check{1}}\right\|_{2} .
$$

In view of (3.1) it would be sufficient to establish the inequalities

and

$$
\left\|u_{N}\right\|_{W_{2}^{L}} \leqq C N^{n / 2-2 L}
$$

$$
\left\|u_{N}\right\|_{2} \leqq C N^{n / 2}
$$

To do this note that any $\xi \in \Omega_{\lambda}, \lambda \in \mathfrak{h}_{\mathfrak{p}_{0}}$, satisfies

$$
|\xi| \leqq C_{1}(1+|\lambda|)^{2}
$$

and also if $|\lambda|$ is large

$$
|\xi| \geqq C_{2}(1+|\lambda|)^{2}
$$


Now since

$$
\left|u_{N}^{(k)}(\xi)\right| \leqq \begin{cases}C N^{-2 k} & \text { if }|\lambda| \leqq N \\ C N^{2 q}(1+|\lambda|)^{-2 q-2 k} & \text { if }|\lambda| \geqq N\end{cases}
$$

where $q$ is an arbitrarily large integer, lemma 3.1 yields

$$
\frac{1}{|c(\lambda)|} \mid\left(\omega^{L}{ }_{u N}\right)^{\hat{(}(\lambda) \mid \leqq} \begin{cases}C N^{-2 L}(1+|\lambda|)^{m / 2} & \text { if }|\lambda| \leqq N \\ C N^{2 q}(1+|\lambda|)^{m / 2-2 q-2 L} & \text { if }|\lambda| \geqq N\end{cases}
$$

It follows that

$$
\begin{aligned}
\left\|u_{N}\right\|_{W_{2}^{L}}^{2} & \leqq C N^{-4 L} \int_{|\lambda| \leqq N}(1+|\lambda|)^{m} d \lambda+C N^{4 q} \int_{|\lambda| \geqq N}(1+|\lambda|)^{m-4 q-4 L} d \lambda \\
& \leqq C N^{m+l-4 L} .
\end{aligned}
$$

This proves the first one of the desired inequalities. The second one is obtained in the same way. Note that $n=m+l, l=\operatorname{dim} \mathfrak{h}_{\mathfrak{p}_{0}}$.

This theorem will now be used to weaken the condition at infinity

THEOREM 3.3. Let $u(z)$ be holomorphic in a p.n. Suppose that $0 \leqq \alpha \leqq \frac{1}{2}$, $\beta>n / 2 \cdot \alpha$ and

$$
\sup _{\text {p.n. }}(1+|z|)^{\beta-k(\alpha-1)}\left|u^{(k)}(z)\right|<\infty
$$

for all $k=0,1, \ldots$

Then $\left\|u_{N}(\chi)\right\|_{m_{p}} \leqq C$ if $N \geqq N_{0}$.

Proof. Let $M$ be a large integer. Put

$$
G(z)=\frac{2}{(M-1) !} z^{M} e^{-z}, \quad G_{t}(z)=G\left(\frac{z}{t^{2}}\right)
$$

and

$$
H(z)=\sum_{k=0}^{M-1} \frac{1}{k !} z^{k} e^{-z}, \quad H_{N}(z)=H\left(\frac{z}{N^{2}}\right) .
$$

Then

$$
1-H_{N}(z)=\int_{N}^{\infty} G_{t}(z) \frac{d t}{t}
$$

and

$$
u_{N}=H_{N} u_{N}+\int_{N}^{\infty} G_{t} u_{N} \frac{d t}{t}
$$


Here the $m_{p}$ norm of $H_{N} u_{N}$ is uniformly bounded by the preceeding theorem. To handle $G_{t} u_{N}$ we shall prove the inequality

$$
\left\|G_{t}(\chi) u_{N}(\chi)\right\|_{W_{2}^{L}} \leqq C^{t n / 2-2 L-2 \beta+4 L \alpha} N^{2 \beta-4 L \alpha} \quad \text { if } t \geqq N \geqq N_{0}
$$

valid also if we replace $L$ by 0 . Then (3.1) yields

$$
\begin{aligned}
\int_{N}^{\infty}\left\|G_{t}(\chi) u_{N}(\chi)\right\|_{m_{p}} \frac{d t}{t} \leqq C N^{2 \beta-n \alpha} \int_{N}^{\infty} t^{n \alpha-2 \beta} \frac{d t}{t}+ \\
+C N^{2 \beta-4 L \alpha} \int_{N}^{\infty} t^{n / 2-2 L-2 \beta+4 L \alpha} \frac{d t}{t} \leqq C+C N^{n / 2-4 L} \leqq C .
\end{aligned}
$$

Thus it only remains to verify the $W_{2}^{L}$ estimate. By assumption we have if $M$ $\geqq k, q$ arbitrary and $\xi \in \Omega_{\lambda}$

$$
\| G_{t}^{(\hat{k})}(\xi) \mid \leqq \begin{cases}C t^{-2 M}(1+|\lambda|)^{2 M-2 k} & \text { if }|\lambda| \leqq t \\ C t^{2 q}(1+|\lambda|)^{-2 q-2 k} & \text { if }|\lambda| \geqq t\end{cases}
$$

and

$$
\left|u_{N}^{(k)}(\xi)\right| \leqq\left\{\begin{array}{ll}
C N^{-2 k} & \text { if }|\lambda| \leqq N \\
C N^{2 \beta-2 k \alpha}(1+|\lambda|)^{-2 \beta+2 k(\alpha-1)} & \text { if }|\lambda| \geqq N
\end{array} .\right.
$$

It follows without difficulty from lemma 3.1 and Leibniz' rule for taking derivatives of a product that

$$
\begin{aligned}
& \frac{1}{|c(\lambda)|}\left|\left(\omega^{L}\left(G_{t} u_{N}\right)\right)^{-}(\lambda)\right| \\
& \quad \leqq \begin{cases}t^{-2 M}(1+|\lambda|)^{m / 2+2 M-2 L} & \text { if }|\lambda| \leqq N \\
t^{-2 M} N^{2 \beta-4 L \alpha}(1+|\lambda|)^{m / 2+2 M-2 L+4 L \alpha-2 \beta} & \text { if } N \leqq|\lambda| \leqq t \\
t^{2 q} N^{2 \beta-4 L \alpha}(1+|\lambda|)^{m / 2-2 q-2 L+4 L \alpha-2 \beta} & \text { if } t \leqq|\lambda| .\end{cases}
\end{aligned}
$$

This implies

$$
\left\|G_{t} u_{N}\right\|_{W_{2}^{L}}^{2} \leqq C t^{-4 M}+C t^{-4 M} N^{n+4 M-4 L}+C t^{n-4 L+8 L \alpha-4 \beta} N^{4 \beta-8 L \alpha} .
$$

If $M$ was choosen sufficiently large, the terms to the right are all less than the last one and the proof is complete.

Remark 3.4. The usual limit for $\beta, \beta>n \alpha|1 / p-1 / 2|$, is obtained by interpolation between the trivial $L_{2}$ case and the above $L_{1}$ result if $p<2$ and by duality if $p>2$.

\section{The compact case.}

Let $U / K$ be a symmetric space of compact type. The elementary spherical functions on $U / K$, denoted by $\Phi_{\Lambda}$, are parametrized by the set of highest 
weights of finite dimensional irreducible representations of $U$ which are of class one with respect to $K$. Let $G / K$ be the noncompact dual of $U / K$ and denote by $\varphi_{\lambda}$ the elementary spherical functions on $G / K$. Then

$$
\begin{aligned}
& \Phi_{\Lambda}(i h)=\underset{-i(\Lambda+\varrho)}{\varphi(h)}, \quad h \in \mathfrak{h}_{\mathfrak{p}_{0}} \\
& \hat{f}(\Lambda)=\int_{U} f(u) \overline{\Phi_{\Lambda}(u)} d u
\end{aligned}
$$

and

$$
\breve{F}(u)=\sum F(\Lambda) \Phi_{\Lambda}(u) d_{\Lambda}
$$

where

$$
d_{\Lambda}=\frac{c(i \varrho) c(-i \varrho)}{c(i(\Lambda+\varrho)) c(-i(\Lambda+\varrho))}
$$

is the dimension of the representation space corresponding to the heighest weight $\Lambda$. Thanks to this close relationship our method of proof for the multiplier theorems applies in the compact case as well. Only condition 2) of theorem 1.4 do not carry over immediately. This condition was used in lemma 3.1 to show that

$$
\left|\frac{c_{j}(\lambda)}{c(\lambda)}\right| \leqq C(1+|\lambda|)^{m / 2}, \quad \lambda \in \mathfrak{h}_{\mathfrak{p}_{0}} .
$$

The analogue of this inequality is however easily proved with the aid of Schur's orthogonality relations

$$
\int_{U} \Phi_{\Lambda_{1}}(u) \overline{\Phi_{\Lambda_{2}}(u)} d u= \begin{cases}0 & \text { if } \Lambda_{1} \neq \Lambda_{2} \\ d_{\Lambda_{1}}^{-1} & \text { if } \Lambda_{1}=\Lambda_{2} .\end{cases}
$$

LEMMA 4.1. In the notation above holds

$$
\left|c_{j}(-i(\Lambda+\varrho))\right| d_{\lambda}^{\frac{1}{2}} \leqq C(1+|\Lambda|)^{m / 2} .
$$

Proof. On one hand

$$
\int_{U} \omega^{L}(u) \Phi_{\Lambda}(u) \overline{\Phi_{\Lambda+\sigma_{j}}(u)} d u=c_{j}(-i(\Lambda+\varrho)) d_{\Lambda+\sigma_{j}}^{-1}
$$

and on the other

$$
\begin{aligned}
\left|\int_{U} \omega^{L}(u) \Phi_{\Lambda}(u) \overline{\Phi_{A+\sigma_{j}}(u)} d u\right| & \leqq \sup _{u \in U}\left|\omega^{L}(u)\right|\left\|\Phi_{\Lambda}\right\|_{2}\left\|\Phi_{\Lambda+\sigma_{j}}\right\|_{2} \\
& \leqq C d_{\Lambda}^{-\frac{1}{2}} d_{\Lambda}^{-\frac{1}{2}} \sigma_{j} .
\end{aligned}
$$


Thus

$$
\left|c_{j}(-i(\Lambda+\varrho))\right| d_{\lambda}^{\frac{1}{2}} \leqq C d_{\lambda}^{\frac{1}{2}}+\sigma_{j} \leqq C(1+|\Lambda|)^{m / 2} .
$$

Since no holomorphy conditions appear in the compact case, functions with compact support can be used, cf. [2]. E.g. one can prove that the Riesz mean operator of order $\alpha$, corresponding to $u(z)=(1-z)_{+}^{\alpha}$, is uniformly bounded on $L_{p}$ if

$$
\alpha>(n-1)\left|\frac{1}{p}-\frac{1}{2}\right| .
$$

\section{REFERENCES}

1. Harish-Chandra, Spherical functions on a semisimple Lie group I, Amer. J. Math. 80 (1958), 241310.

2. L. Vretare, On $L_{p}$ Fourier multipliers on a compact Lie group, Math. Scand. 35 (1974), 49-55.

3. L. Vretare, On $L_{p}$ Fourier multipliers on certain symmetric spaces, Math. Scand. 37 (1975), $111-$ 121.

4. L. Vretare, Elementary spherical functions on symmetric spaces, Math. Scand. 39 (1976), 343-358.

5. G. Warner, Harmonic analysis on semisimple Lie groups II (Grundlehren Math. Wissensch. 189), Springer-Verlag, Berlin, Heidelberg, New York, 1972.

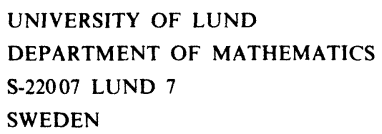

